\title{
Author Correction: Lipid management in patients with chronic kidney disease
}

Charles J. Ferro, Patrick B. Mark, Mehmet Kanbay, Pantelis Sarafidis, Gunnar H. Heine, Patrick Rossignol, Ziad A. Massy, Francesca Mallamaci, Jose M. Valdivielso, Jolanta Malyszko, Marianne C. Verhaar, Robert Ekart, Raymond Vanholder, Gerard London, Alberto Ortiz and Carmine Zoccali

Nature Reviews Nephrology (2018) https://doi.org/10.1038/s41581-018-0072-9

Published online 25 October 2018

In the acknowledgements section of this article as originally published, information on the authors' roles as EURECAm members is missing. The correct acknowledgement is as follows: "This Review was planned as part of the activity of the European Renal and Cardiovascular Medicine working (EURECAm) group and all authors are EURECAm members. A.O.'s work was supported by Spanish Government ISCIII FEDER funds (PI16/02057, ISCIII-RETIC REDinREN RD16/0009) and Community of Madrid (B2017/BMD-3686 CIFRA2-CM). P.R.'s work is supported by a public grant overseen by the French National Research Agency (ANR) as part of the second "Investissements d'Avenir" program FIGHT-HF (reference: ANR-15-RHU-0004) and by the French PIA project “Lorraine Université d'Excellence”, reference ANR-15-IDEX-04-LUE.” The omission has been corrected in the PDF and HTML versions of the article.

https://doi.org/10.1038/s41581-018-0099-y I Published online 11 December 2018

\section{Author Correction: Estimated GFR: time for a critical appraisal}

Esteban Porrini, Piero Ruggenenti, Sergio Luis-Lima, Fabiola Carrara, Alejandro Jiménez, Aiko P. J. de Vries, Armando Torres, Flavio Gaspari and Giuseppe Remuzzi

Nature Reviews Nephrology (2018) https://doi.org/10.1038/s41581-018-0080-9 Published online 05 December 2018

In the version of this article originally published online, the middle initials of Aiko P. J. de Vries, an author on the manuscript, were omitted. The omission has been corrected in the PDF and HTML versions of the article.

https://doi.org/10.1038/s41581-018-0105-4 I Published online 18 December 2018

\section{Publisher Correction: The evaluation of monoclonal gammopathy of renal significance: a consensus report of the International Kidney and Monoclonal Gammopathy Research Group}

Nelson Leung, Frank Bridoux, Vecihi Batuman, Aristeidis Chaidos, Paul Cockwell, Vivette D. D’Agati, Angela Dispenzieri, Fernando C. Fervenza, Jean- Paul Fermand, Simon Gibbs, Julian D. Gillmore, Guillermo A. Herrera, Arnaud Jaccard, Dragan Jevremovic, Efstathios Kastritis, Vishal Kukreti, Robert A. Kyle, Helen J. Lachmann, Christopher P. Larsen, Heinz Ludwig, Glen S. Markowitz, Giampaolo Merlini, Peter Mollee, Maria M. Picken, Vincent S. Rajkumar, Virginie Royal, Paul W. Sanders, Sanjeev Sethi, Christopher P. Venner, Peter M. Voorhees, Ashutosh D. Wechalekar, Brendan M. Weiss and Samih H. Nasr 